\title{
Geomagnetically induced currents in Europe
}

\section{Modelled occurrence in a continent-wide power grid}

\author{
Ari Viljanen ${ }^{1, *}$, Risto Pirjola ${ }^{1,2}$, Ernö Prácser ${ }^{3}$, Juri Katkalov ${ }^{4}$, and Magnus Wik ${ }^{5}$ \\ 1 Finnish Meteorological Institute, Erik Palmenin aukio 1, 00560 Helsinki, Finland \\ ${ }^{*}$ Corresponding author: ari.viljanen@fmi.fi \\ 2 Natural Resources Canada, Geomagnetic Laboratory, 2617 Anderson Road, Ottawa, ON, K1A 0E7, Canada \\ 3 Geodetic and Geophysical Institute, RCAES, HAS, Csatkai Endre Utca 6-8, 9400 Sopron, Hungary \\ 4 Polar Geophysical Institute, Fersman St. 14, 184209 Apatity, Murmansk Region, Russia \\ 5 NeuroSpace, Lotsgatan 33B, 21642 Limhamn, Sweden
}

Received 5 November 2013 / Accepted 23 February 2014

\begin{abstract}
Statistics of geomagnetically induced currents (GIC) in the European high-voltage power grids based on 1-min geomagnetic recordings in 1996-2008 and on 1-D models of the ground conductivity have been derived in the EURISGIC project (European Risk from Geomagnetically Induced Currents). The simplified yet realistic power grid model indicates that large GIC can occur anywhere in Europe. However, geomagnetic variations are clearly larger in North Europe, so it is the likely region of significant GIC events. Additionally, there are areas in the North with especially low ground conductivities, which further tend to increase GIC. The largest modelled GIC values at single substations in 1996-2008 are about $400 \mathrm{~A}$ in the Nordic Countries, about $100 \mathrm{~A}$ in the British Isles, about $80 \mathrm{~A}$ in the Baltic Countries, and less than $50 \mathrm{~A}$ in Central and South Europe. The largest GIC event in the period studied is the Halloween storm on 29-30 October 2003, and the next largest ones occurred on 15 July 2000 and 9 November 2004.
\end{abstract}

Key word. geomagnetically induced currents (GIC)

\section{Introduction}

The EURISGIC (European Risk from Geomagnetically Induced Currents) project has applied previous methods to calculate geomagnetically induced currents (GIC) at a continental scale (Viljanen 2011; Viljanen et al. 2012). One objective of the EURISGIC project is to derive a map showing the statistical occurrence of GIC throughout Europe. This helps power companies to assess a possible GIC risk and to analyse past events which possibly have affected power transmission.

In a recent paper, Viljanen et al. (2013) considered the model of the Finnish $400 \mathrm{kV}$ power grid in 1978-1979. Using the ground conductivity models by Ádám et al. (2012) and geomagnetic data of the active year 2003, they calculated GIC in the grid when moved across Europe. This made it possible to present a map of relative GIC amplitudes at different locations in Europe. Because the precise parameters of the Finnish grid of 1978-1979 are known, the map provides a quantitative reference model. Viljanen et al. (2013) also discussed the sensitivity of the results with respect to variations of the grid parameters.

In this paper, we consider GIC in the European high-voltage power grids (220 kV and above) during a full sunspot cycle in 1996-2008. We first briefly summarize the methods, after which we present characteristic spatial and temporal features of GIC.

\section{Methods}

Viljanen et al. (2012) provide a recent comprehensive overview of modelling GIC in a large power grid. We will not repeat the details here, but concentrate on the most relevant points concerning input data and output quantities.

The first step is to calculate the geoelectric field at Earth's surface. For this, we use geomagnetic recordings as 1-min time series from European observatories and other measurement sites in 1996-2008. We relate the local magnetic field to the electric field by the surface impedance determined by the local 1-D ground conductivity model given by Ádám et al. (2012). We consider two cases here: a single 1-D ground model applied everywhere, and the full conductivity map with several different blocks. The former illustrates how the spatial variability of geomagnetic variations affects the electric field. The latter also takes into account the variability due to the ground itself.

We consider GIC in a prototype grid model covering Europe in four separate parts: (1) South and Central Europe; (2) Nordic countries (including North-West Russia); (3) British Isles (UK and Ireland); (4) Baltic countries (see Fig. 1 for a schematic map). As discussed by Viljanen et al. (2012), the power grid model includes approximations and thus provides only indicative measures for GIC.

The effect of the topology of the grid on GIC is illustrated in Figure 1. Assuming a spatially uniform electric field reveals the sites at which large GIC are possible. A general finding is that nodes at the edges of the grid are such locations. Additionally, there are corner points or end points of lines in the middle parts of the grid that can also experience large currents. As Figure 1 shows, nearly equally large GIC can, in principle, occur in any part of the European grid. 

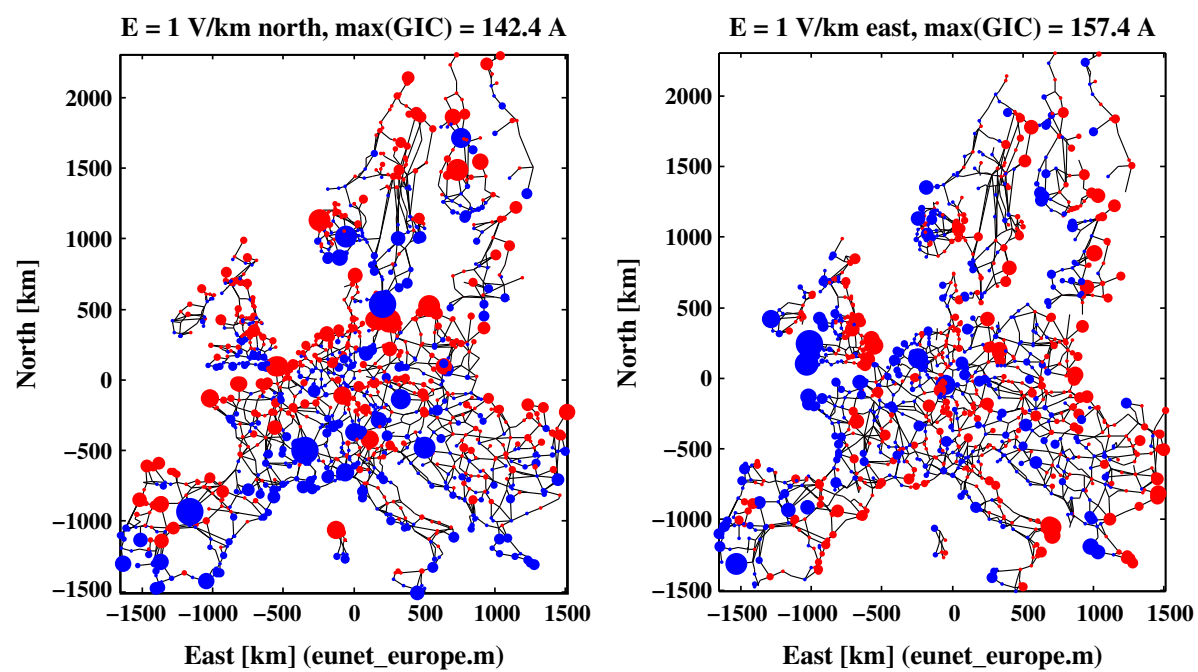

Fig. 1. GIC in the European high-voltage grid due to a spatially constant electric field of $1 \mathrm{~V} / \mathrm{km}$ pointing to the North (left plot) or to the East (right plot). Red denotes currents flowing from the grid into the ground and blue denotes currents flowing from the ground into the grid.

The basic output of the GIC software is a 1-min time series of GIC values at each node of the power grid. The total number of 1-min time steps in 1996-2008 is about 6.8 million. All values are not routinely saved, but information is summarized by the following quantities at each node. In this paper, GIC refers to the sum of the induced currents in the three phases of a power system. We consider only the absolute values, since they are relevant concerning possible effects on transformers.

- Hourly maximum.

- Daily maximum and its occurrence time.

- Daily averages.

- Hourly averages.

- Histogram data (number of values for each selected bin).

Additionally, we save the sum of GIC of all nodes (substations) as 1-min values.

\section{Results}

\subsection{Electric field}

We used a ground conductivity model of Europe consisting of blocks with a layered structure (Ádám et al. 2012). The full block conductivity model is available at http://real.mtak.hu/ 2957/ and reproduced as additional material to our paper. Figure 2 shows the blocks and the conductances calculated by integrating the conductivity from the surface down to $80 \mathrm{~km}$. This map indicates qualitatively the expected magnitudes of the electric field. If the magnetic variation field is identical everywhere then the electric field is larger in blue areas with smaller conductivities in the top ground layers. Outside of the blocks shown in the figure, we assume a simple 2-layer ground model (Viljanen et al. 2006), whose upper layer is $150 \mathrm{~km}$ and resistivity is $38.5 \Omega \mathrm{m}$. The lower infinite layer has the resistivity of $0.385 \Omega \mathrm{m}$. However, the exact choice of this model is irrelevant, since these areas are outside of the power grid.

We also considered a simplified test case assuming a single layered model of the ground conductivity everywhere (Table 1). The selected model represents a region mostly in Lithuania in

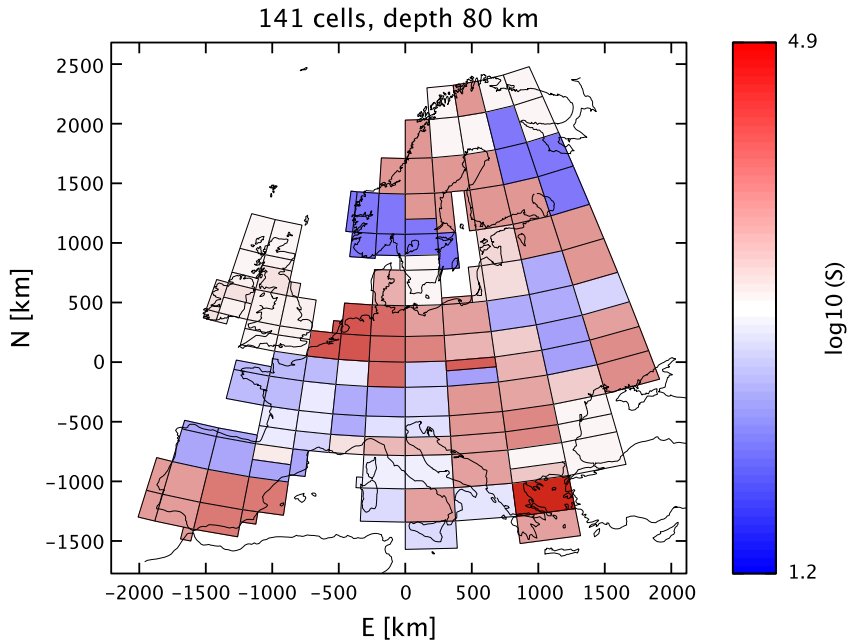

Fig. 2. Conductances all over Europe obtained by the integration of the conductivity down to the depth of $80 \mathrm{~km}$. Figure based on Ádám et al. (2012). Numerical values are available at http://real.mtak.hu/ $2957 /$.

Table 1. Thicknesses and resistivities of the layers in the ground model applied for whole Europe in a test case.

\begin{tabular}{cc}
\hline \hline Thickness $(\mathrm{km})$ & Resistivity $(\Omega \mathrm{m})$ \\
\hline 0.4 & 40 \\
1.3 & 3 \\
140.0 & 2000 \\
170.0 & 118 \\
$\infty$ & 15 \\
\hline
\end{tabular}

the latitude range of $54.5-57.2 \mathrm{~N}$ and longitude range of 20.0-26.7 E. The related conductance represents a typical value of the full conductivity map in Figure 2, as shown by Viljanen et al. (2013).

We calculated the maximum value of the electric field on each day in 1996-2008 in a regular surface grid covering the area in Figure 2. The medians of these values are shown in Figure 3. The left-hand-side plot in Figure 3 shows the obvious 

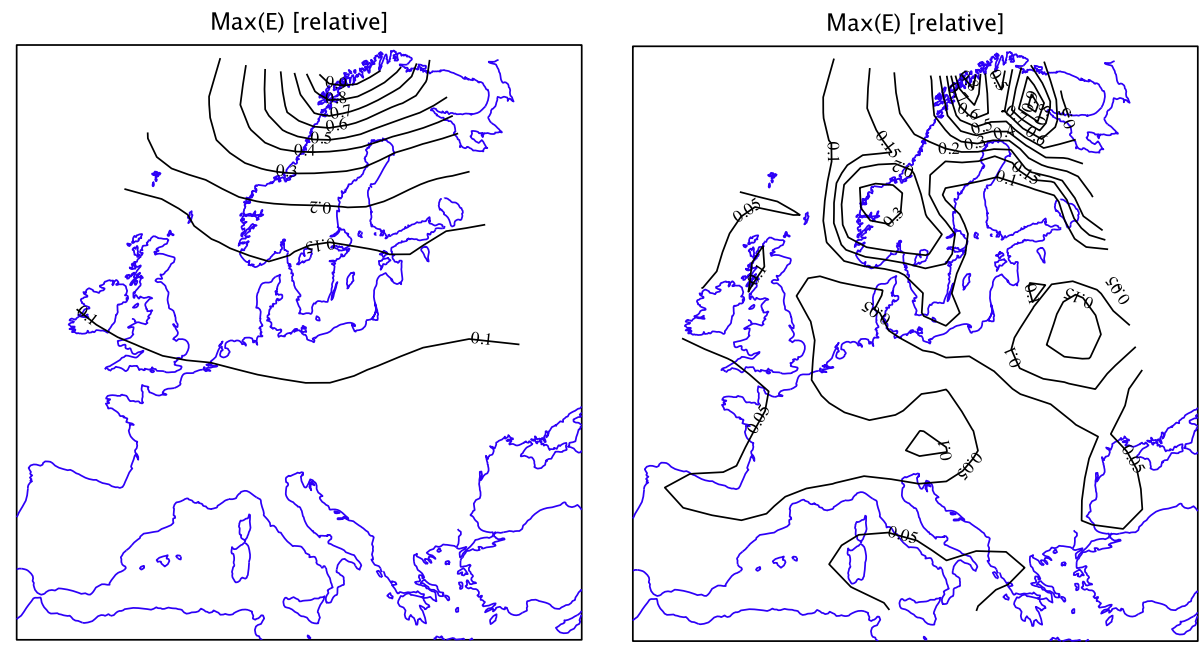

Fig. 3. Left: median of the daily maximum values of the electric field in 1996-2008 using the conductivity Model 01 of Table 1 and a regular surface grid covering the area in Figure 2. Right: as in the left, but using the full conductivity map shown in Figure 2. In both plots, the largest value is normalized to unity.

feature that larger geomagnetic variations at high latitudes also cause larger electric fields there. The right-hand-side plot in Figure 3 has a more complicated structure, because the variations of the ground conductivity are mixed with the spatial variability of the geomagnetic field. In both cases, the electric field has its largest values in North Europe at geographic latitudes higher than about 55 degrees. We also note that the sharp decrease of the electric field in the North-West corner is due to the lack of geomagnetic recordings there. The interpolated magnetic field at that region approaches zero when the distance from observatories increase.

\subsection{Occurrence of GIC in the European grids}

As already mentioned, we calculated GIC as 1-min series at all nodes of the prototype power grid model. Because this model is an approximation, we prefer to present geographically averaged results. We illustrate the idea first for the Finnish grid. The lefthand-side plot in Figure 4 shows the maximum GIC in 1996-2008 at single substations. For averaging these results, we set a regular grid on the map, and for each grid point, we consider the nearest four substations. The GIC value given for the grid point is the mean value of the maximum GIC at the four substations. As the right-hand-side plot in Figure 4 shows, such a smoothing provides a reasonable regional indicator of GIC magnitudes. Due to the averaging method, the maximum GIC is smaller than at any single substation.

The result for whole Europe is shown in the upper plot of Figure 5. The maximum GIC are much larger in North Europe than elsewhere, as expected based on the electric field distribution in Figure 3. The British Isles and Baltic Countries also have larger GIC than Central and South Europe. For clarity, we present Central and South Europe separately in the lower plot of Figure 5. There is a cluster of "hot spots" in the middle parts of the grid in a zone from France to Hungary. This region has a small conductance as shown in Figure 2, so an enhancement of the electric fields and GIC can be expected there. Consequently, we note the fairly notable effect of the ground conductivity on the amplitudes of GIC. If we assume the single conductivity model of Table 1 , thus ignoring spatial variations due to a nonuniform ground, then the maximum sites are concentrated in the coastal area from France to Germany (figure not
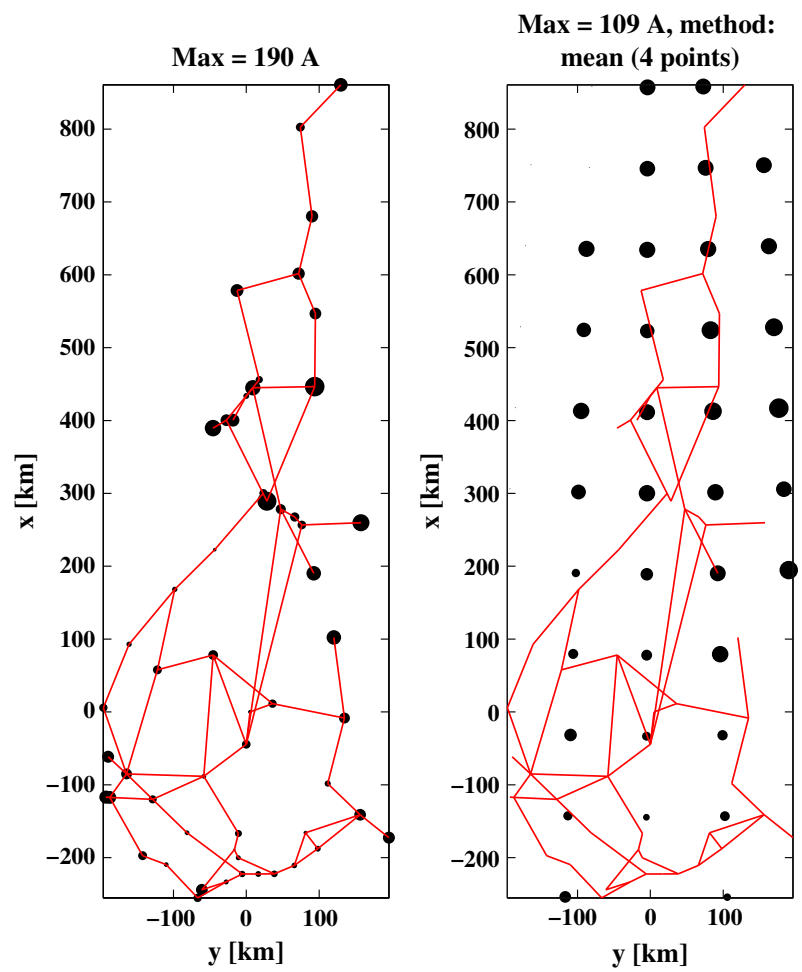

Fig. 4. Left: maximum of the modelled GIC at the substations of the Finnish high-voltage power grid in 1996-2008. Right: regionally averaged presentation of the maximum GIC. The scale of the dots is different in the plots.

shown). This is clear, because geomagnetic variations are large there causing large electric fields, and there are also several edge nodes which are prone to GIC (cf. Fig. 1).

Table 2 gives explicit values of the largest modelled GIC at single substations in different parts of Europe. They are consistent with the characteristics of the geoelectric field (Fig. 3). Nordic countries experience clearly the largest GIC, whereas in South and Central Europe the maxima are about one decade smaller. We emphasize that the values in Table 2 are only indicative due to uncertainties in the power grid and ground 

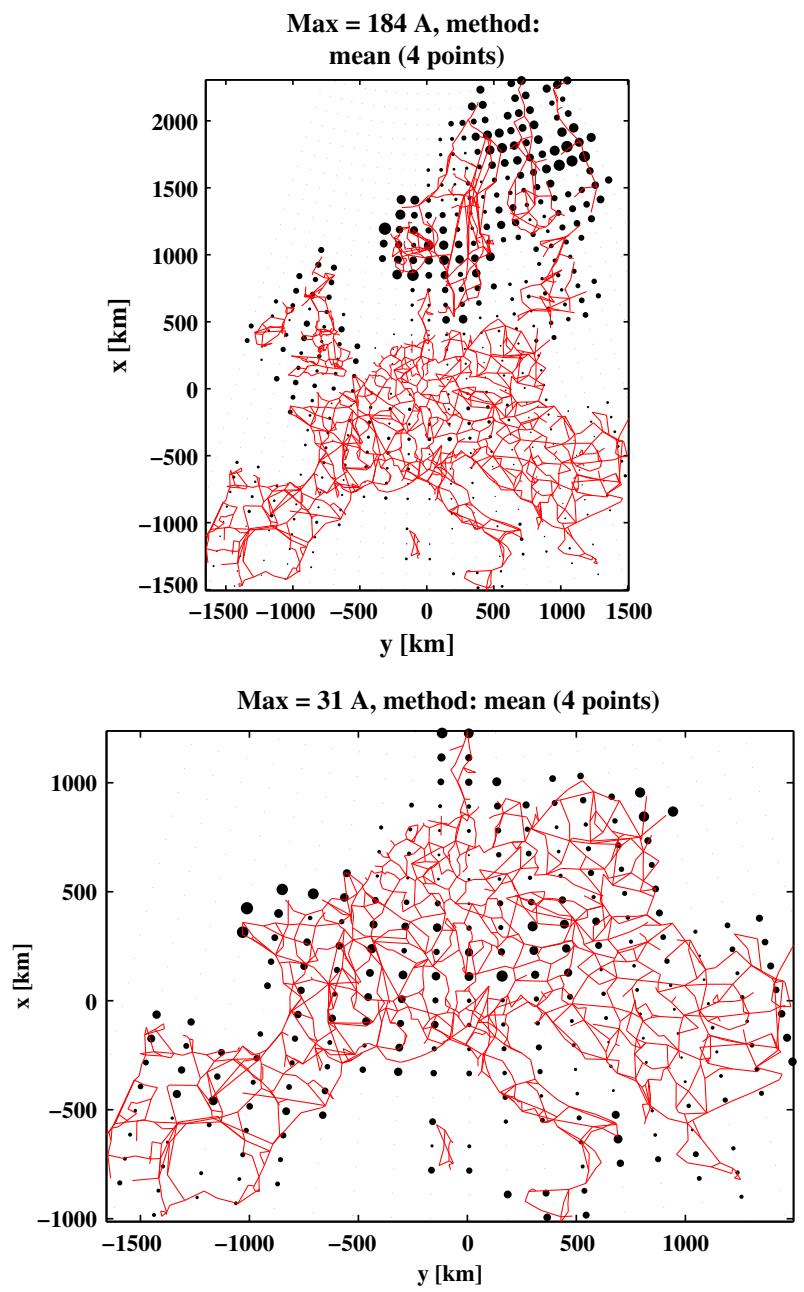

Fig. 5. Top: regionally averaged maximum of the modelled GIC at the substations of the European high-voltage power grid in 19962008. Bottom: separate presentation for Central and South Europe. For clarity, the scales are different in the figures.

Table 2. Maximum GIC A (top 1), the 10th largest maximum (top 10), average of the maximum GIC and median of the maximum GIC at the substations in different parts of the European high-voltage power grid. Continent refers to South and Central Europe.

\begin{tabular}{crrrr}
\hline \hline Region & Top 1 & Top 10 & Max & Median \\
\hline Nordic & 427.0 & 184.3 & 56.6 & 41.8 \\
Baltic & 77.7 & 39.5 & 23.9 & 18.1 \\
British Isles & 96.5 & 57.8 & 20.5 & 15.2 \\
Continent & 50.0 & 32.7 & 7.5 & 5.2 \\
All & 427.0 & 184.3 & 19.3 & 9.2 \\
\hline
\end{tabular}

conductivity models. Beggan et al. (2013) have recently estimated extreme GIC in the UK. For a return period of once per 30 years, they find in their Table 4 the maximum GIC to be a little larger than $100 \mathrm{~A}$. This is comparable to our estimate of the maximum of $100 \mathrm{~A}$ in 1996-2008. As a side note, Beggan et al. (2013) estimate that the largest GIC occurring in the UK once in 100 years could reach about $300 \mathrm{~A}$ and even exceed 500 A once in 200 years.

For comparison, Table 3 shows the result when assuming a single ground conductivity model given in Table 1 everywhere. The Nordic countries still have the largest GIC values, although
Table 3. As Table 2 but assuming a single ground conductivity model of Table 1 everywhere.

\begin{tabular}{crccc}
\hline \hline Region & Top 1 & Top 10 & Max & Median \\
\hline Nordic & 184.1 & 107.7 & 35.0 & 26.6 \\
Baltic & 88.4 & 26.5 & 21.2 & 16.6 \\
British Isles & 68.4 & 43.6 & 16.3 & 12.6 \\
Continent & 94.3 & 45.3 & 9.7 & 7.3 \\
All & 184.1 & 107.7 & 15.9 & 9.9 \\
\hline
\end{tabular}

Table 4. Most active GIC days in the European high-voltage power grid according to the maximum 1-min sum of GIC of all nodes divided by the number of nodes.

\begin{tabular}{cccccc}
\hline \hline & Day & GIC A & & Day & GIC A \\
\hline 01 & 20031030 & 10.0 & 21 & 20031014 & 3.1 \\
02 & 20031029 & 9.3 & 22 & 19980924 & 3.1 \\
03 & 20000715 & 7.5 & 23 & 20030529 & 3.1 \\
04 & 20041109 & 5.8 & 24 & 20020907 & 3.0 \\
05 & 20011106 & 4.8 & 25 & 20041107 & 2.9 \\
06 & 20031031 & 4.8 & 26 & 20020523 & 2.8 \\
07 & 20000406 & 4.8 & 27 & 19990922 & 2.8 \\
08 & 20041108 & 4.2 & 28 & 19980827 & 2.8 \\
09 & 20021001 & 3.9 & 29 & 20011022 & 2.8 \\
10 & 20011124 & 3.9 & 30 & 20010413 & 2.8 \\
11 & 20010331 & 3.8 & 31 & 19990730 & 2.7 \\
12 & 20050911 & 3.7 & 32 & 20000918 & 2.7 \\
13 & 20050824 & 3.7 & 33 & 20050508 & 2.6 \\
14 & 19980504 & 3.5 & 34 & 20050107 & 2.6 \\
15 & 20000917 & 3.5 & 35 & 20051211 & 2.5 \\
16 & 20031120 & 3.5 & 36 & 19990113 & 2.4 \\
17 & 20050121 & 3.2 & 37 & 20031122 & 2.4 \\
18 & 20040726 & 3.2 & 38 & 20061215 & 2.4 \\
19 & 20050515 & 3.2 & 39 & 20011012 & 2.4 \\
20 & 20000524 & 3.2 & 40 & 20040727 & 2.4 \\
\hline
\end{tabular}

the difference to the other parts of Europe is smaller than in Table 2. This is due to the larger conductivity in the Nordic Countries when using the ground model of Table 3 instead of the full conductivity map. The increase of the maximum GIC in the continental Europe follows from the lower conductivities especially in Denmark and in the North-West parts of continent. Because geomagnetic variations are largest there and these parts are at the edge of the grid, GIC tend to increase there. On the other hand, regions in the middle part of the continent now have a larger conductivity and smaller GIC.

Table 4 shows the list of the most active GIC days. The activity measure used here is the largest daily sum of GIC at all nodes as calculated from 1-min values. Division of the sum by the number of nodes gives a meaningful scaling. Most of the top events occur after the year 2000 during the descending phase of the sunspot cycle 23. The Halloween storm on 29-30 October 2003 is definitely the largest event in the European scale. To see whether the ranking of storms changes significantly depending on the geographic region, we also considered separately the four parts of the European grid. Then the top days were 29 or 30 October 2003, except for South and Central Europe where 15 July 2000 is the first one being slightly more active than 29-30 October 2003.

Besides looking at daily maximum values, it is appropriate to measure activity by studying 1-min values too. We sorted the 
A. Viljanen et al.: Geomagnetically induced currents in Europe
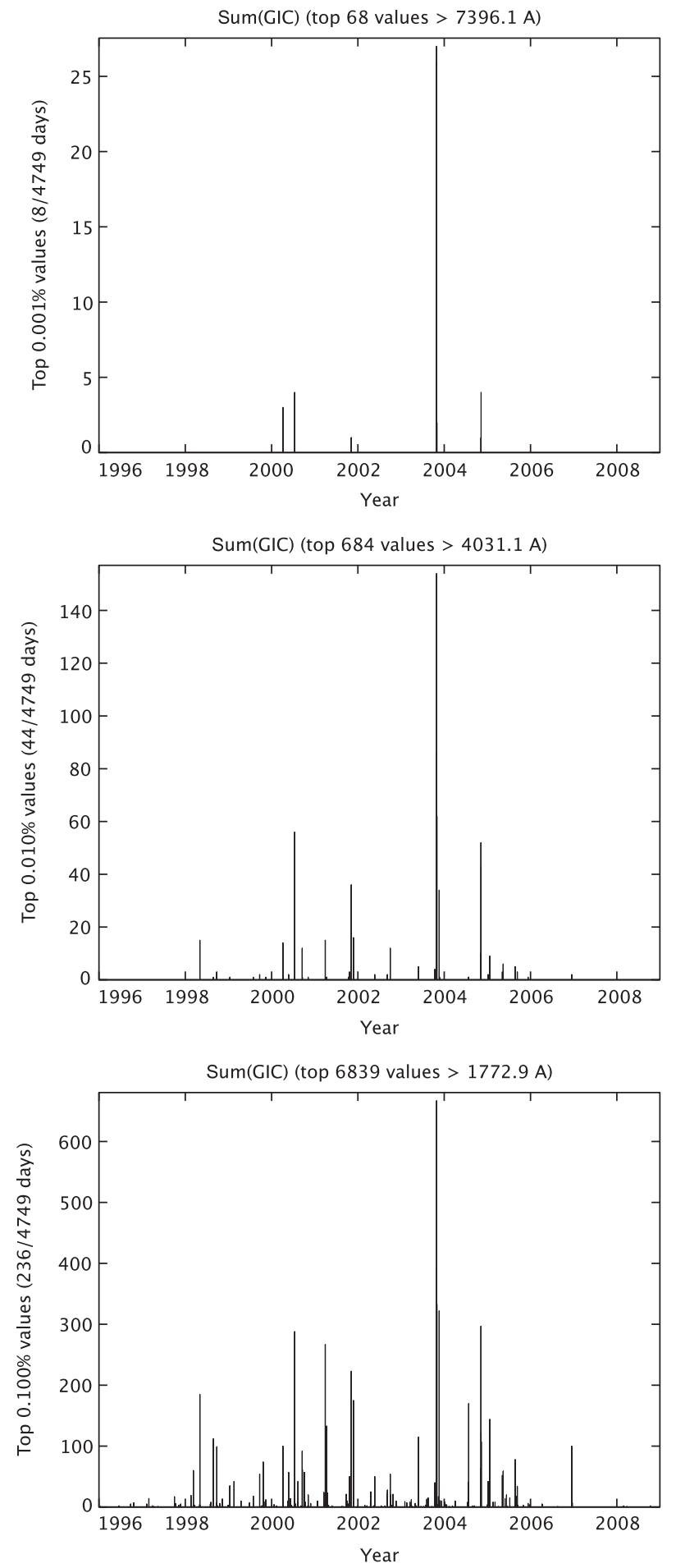

Fig. 6. Days of large GIC as ranked by the sum of absolute GIC of all nodes in the European high-voltage grid (number of large 1-min values shown). From top to bottom panel: occurrence of the top $0.001 \%, 0.01 \%, 0.1 \%$ of all values.

full time series of the sum of GIC and counted the daily occurrence of large values (Fig. 6). By "large", we refer to values exceeding the threshold for a given quantile of $0.001 \%$, $0.01 \%$ and $0.1 \%$ of the top values. Such a ranking demonstrates even more clearly that the 29-30 October 2003 storm was the most dominating in 1996-2008. This holds for the separate parts of the European grid too.
The top 1-min values of the $0.001 \%$ quantile occurred on eight different days in 1996-2008, which roughly means one day every 1.5 years on average. However, there were such days only in 2000-2001 and 2003-2004 in the 13-year period. The top $0.01 \%$ quantile corresponds to $3-4$ days per year, or to an active day about every 3rd or 4th month. The lowest category of the top $0.1 \%$ cases is quite common occurring once or twice every month. However, these events are not uniformly distributed either, but there are months below this activity level. Especially, 2007-2008 were nearly completely quiet in this sense (cf. Minamoto \& Taguchi 2009).

The seasonal distribution of large GIC is shown in Figure 7. Again, the dominance of the October 2003 storm is very strong causing apparent bias. To see this, we made a test by excluding 29-31 October 2003. Then the activity in October decreases to about the same level as in September. However, there is now a prominent maximum in November 2003, as could expected from Figure 6 too. Consequently, the time series of 1996-2008 does not produce a clean seasonal variation of top GIC values.

The diurnal distribution (Fig. 8) has also certain features due to the 29-30 October 2003 storm. Especially for the top $0.001 \%$ values, there is high activity in 7-8 UT and in 19-21 UT. This morning maximum is due to the active time on 29 October 2003 related to the large sudden impulse and consequent variations at the beginning of the storm. The evening maximum is associated with rapid variations of the strong westward electrojet on 30 October 2003, which caused the blackout in Malmö in southern Sweden (Pulkkinen et al. 2005). When considering the $0.1 \%$ top values, the diurnal distribution becomes more regular with a quiet time close to noon and an evening maximum followed by quite high activity in the morning. This is consistent at least with the high-latitude behaviour of the time derivative of the magnetic field with intensifications due to the evening westward electrojet and morning pulsations (Viljanen et al. 2001; Weigel et al. 2003; Viljanen \& Tanskanen 2011). We remark that a detailed study of the diurnal behaviour should be done more locally to consider better the magnetic local time instead of UT.

Distributions of the continuous sequences of a large sum of GIC are shown in Figure 9. They typically last only a few minutes, although there are some much longer events. For the European grid, the longest sequence occurs on 6 November 2001 with all thresholds (Fig. 10 shows the case of top $0.1 \%$ values).

\section{Discussion}

Estimations of the maximum GIC given in Table 2 and the list of the largest events in Table 4 get some independent support from European GIC recordings. In 1996-2008, the Fingrid Oyj power company has performed measurements at three transformer neutrals in Finland (Pirttikoski, Rauma, Yllikkälä). The largest value was about $40 \mathrm{~A}$ at Pirttikoski (30 October 2003), 42 A at Rauma (30 October 2003) and 27 A at Yllikkälä (15 July 2000). The true resistances met by GIC at the transformer stations are larger (1-3 ohm) than assumed in the prototype grid model $(0.5 \mathrm{ohm}$ everywhere). We can then roughly estimate that $100 \mathrm{~A}$ could have reached with smaller resistances.

Recordings at the Finnish natural gas pipeline give additional information. Although the pipeline is different from the power grid, GIC along the pipeline reaches comparable 

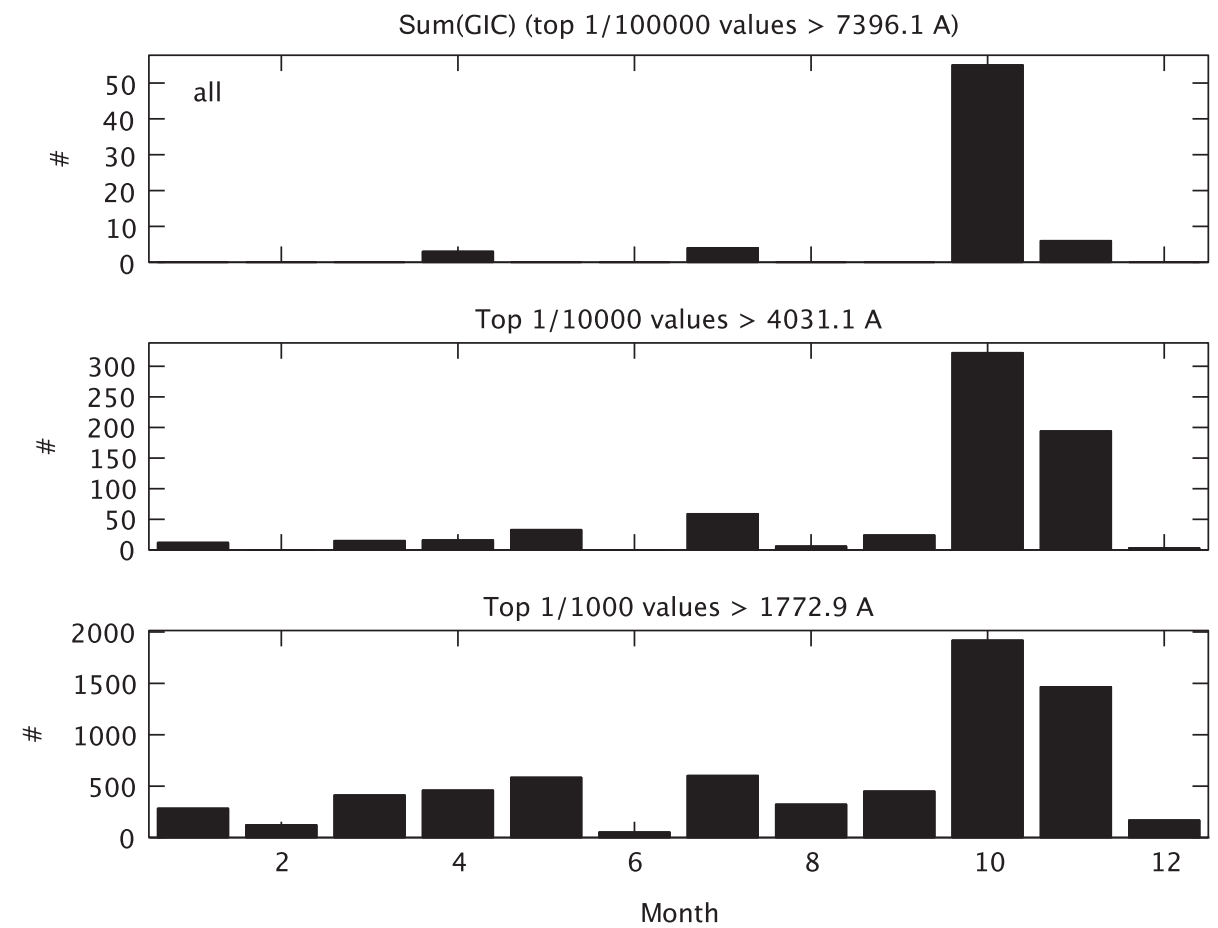

Fig. 7. Seasonal distribution of the largest 1-min GIC sums in the European high-voltage power grid. From top to bottom: occurrence of the top $0.001 \%, 0.01 \%, 0.1 \%$ of all values.
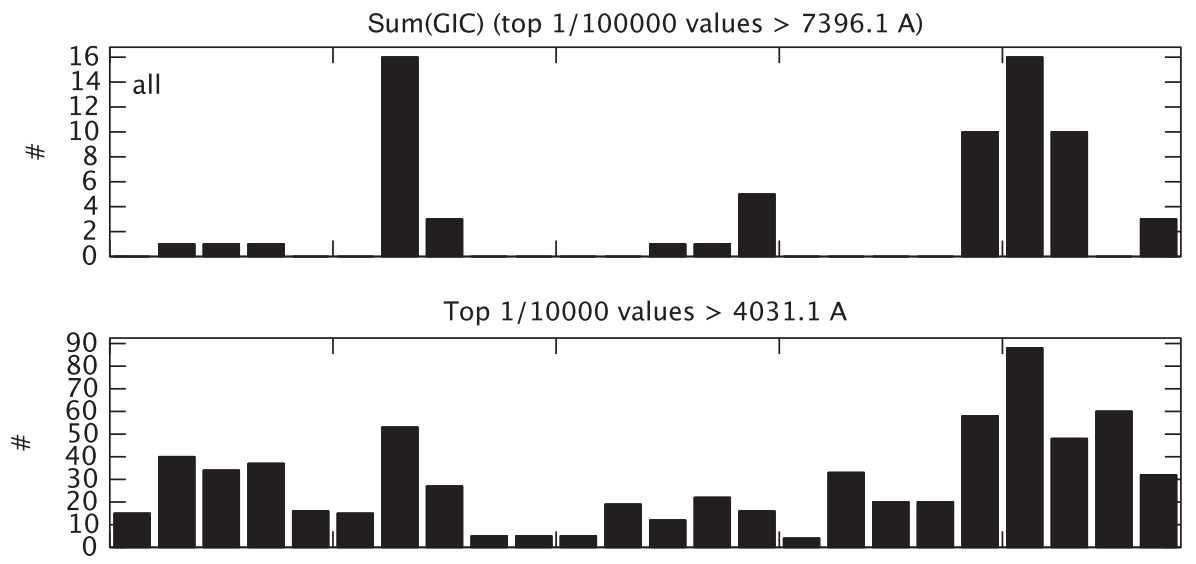

Top $1 / 1000$ values $>1772.9 \mathrm{~A}$

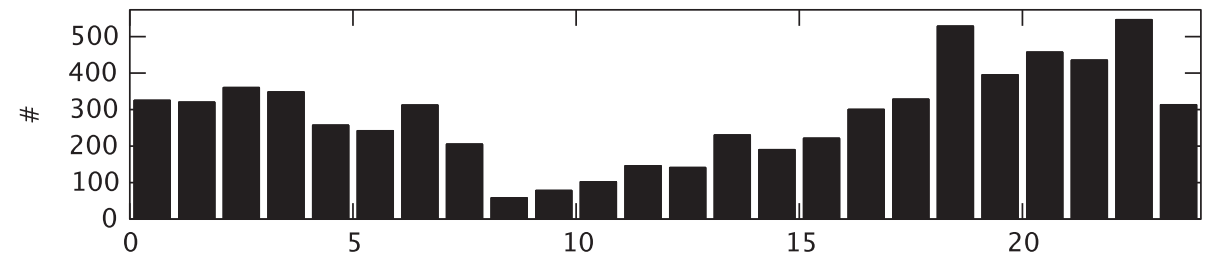

UT [h]

Fig. 8. Diurnal distribution of the largest 1-min GIC sums in the European high-voltage power grid. From top to bottom: occurrence of the top $0.001 \%, 0.01 \%, 0.1 \%$ of all values.

magnitudes. The largest pipeline GIC during the study period is 57 A on 29 October 2003 (Viljanen et al. 2010).

The largest GIC measured at a transformer in southern Sweden are 269 A on 6 April 2000, 222 A on 15 July 2000 (Wik et al. 2008) and 200 A on 30 October 2003 (Wik et al. 2009). In Scotland, the peak value has been about $40 \mathrm{~A}$ on 30 October 2003 (Thomson et al. 2005). A recent study by
Myllys et al. (2014) using precise parameters of the Norwegian power grid gives consistent results with the simplified EURISGIC grid model.

The number of available GIC recordings from Central and South Europe is very small. Torta et al. (2012) have recently modelled GIC in a small part of the Spanish grid. For the event of 24-25 October 2011 they show a measurement of about 2 A. 
A. Viljanen et al.: Geomagnetically induced currents in Europe

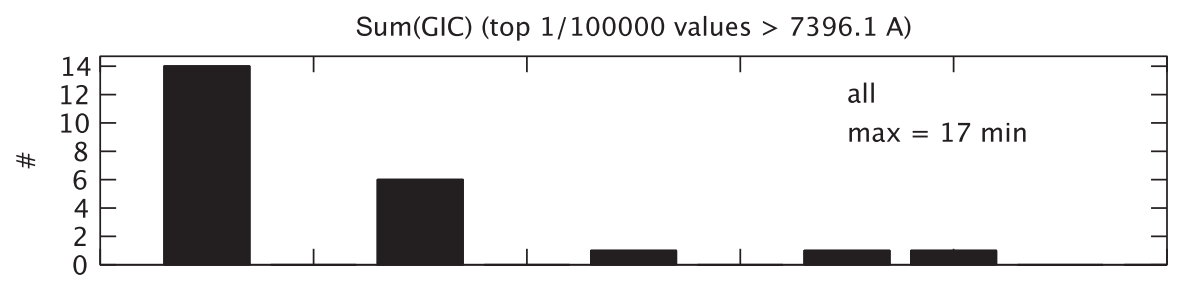

Top $1 / 10000$ values $>4031.1 \mathrm{~A}$

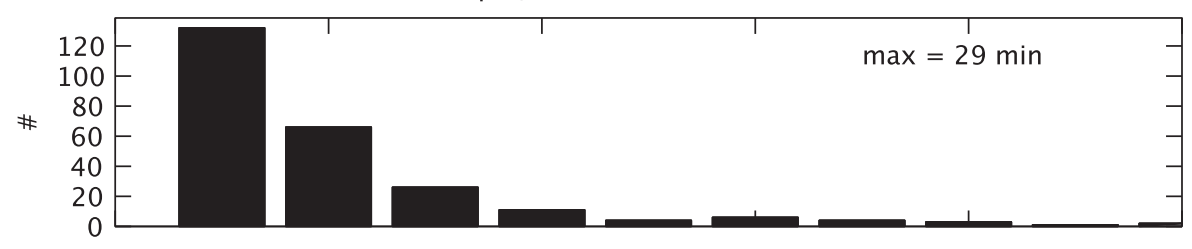

Top $1 / 1000$ values $>1772.9 \mathrm{~A}$

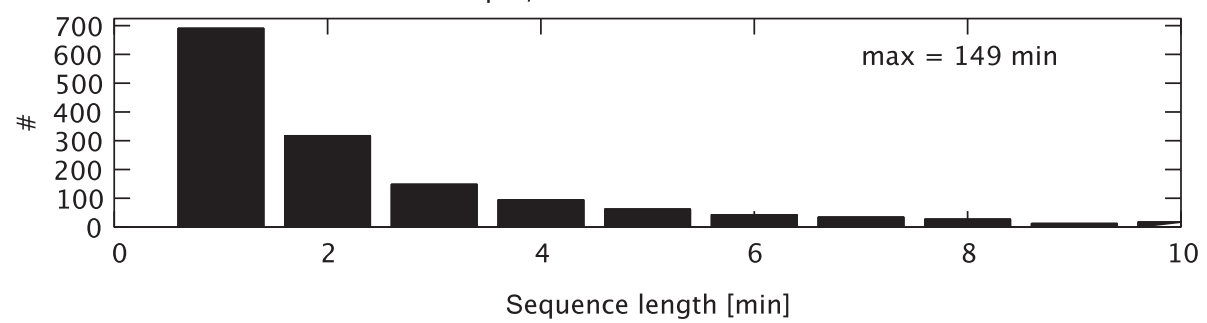

Fig. 9. Distribution of continuous sequences of the largest 1-min GIC sums in the European high-voltage power grid. From top to bottom: occurrence of the top $0.001 \%, 0.01 \%, 0.1 \%$ of all values. The maximum length of the sequences corresponding to each activity category is also given in the subplots.

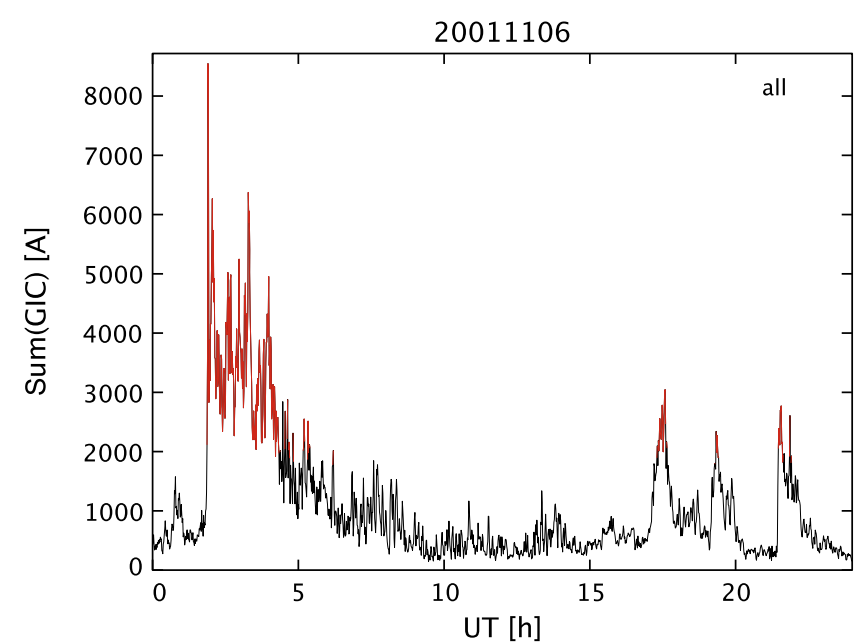

Fig. 10. Sum of GIC in the European high-voltage power grid on 6 November 2011. Red colour denotes periods when the sum exceeds the limit for the top $0.1 \%$ values (cf. Fig. 9). The longest sequence of large GIC lasts for $149 \mathrm{~min}$.

Although this is a rather quiet event, they could use it to estimate GIC during a severe storm of 24 March 1991 indicating a current of about $33 \mathrm{~A}$ at one substation. This supports our estimations that tens of amperes are possible during the largest storms in South and Central Europe.

We also note that GIC up to 76 A (9 November 2004) have been reported in China (Liu et al. 2009), which corresponds to South Europe regarding geomagnetic latitudes. When comparing the Chinese results to Europe, we must take into account that there are long transmission lines up to $300 \mathrm{~km}$ in China, which favours the existence of larger GIC than in Europe with clearly shorter lines. There are also recordings and estimations of GIC in Japan indicating the possibility of currents up to about 20-25 A (Watari et al. 2009; Pulkkinen et al. 2010).

Studies in the southern hemisphere show that the same storms have caused the major events there as in the North. Examples are available from Australia (Marshall et al. 2011), New Zealand (Marshall et al. 2012), South Africa (Ngwira et al. 2008) and Brazil (Trivedi et al. 2007).

\section{Conclusions}

There are three factors contributing to the occurrence of GIC: topology of the power grid, geomagnetic variations, and the ground conductivity. The prototype grid model used in this study indicates that all areas in Europe can in principle experience large GIC if the electric field has no spatial variations. However, the spatial variability of both the magnetic field and the ground conductivity create strongly nonuniform electric fields.

As is well-known, the geomagnetic field and its time derivative are generally larger in the north close to the auroral region than in South and Central Europe. If the ground conductivity were identical everywhere then the electric field would decrease smoothly towards the south on average. The true conductivity is much more complex, which causes an additional spatial variation. However, North Europe is still the most likely area of large electric fields. Especially, there are regions in the North having a small ground conductivity, which tends to further increase the electric field. There are similar regions in Central Europe producing local maxima of the electric field, although the magnitudes remain clearly smaller than in the North.

Based on geomagnetic data of 1996-2008 and the ground conductivity model consisting of 1-D blocks, GIC levels are highest in the Nordic countries (maximum up to a few hundred 
amperes as 1-min values) and clearly smaller in South and Central Europe (less than $50 \mathrm{~A}$ ). However, we emphasize that the amplitude of GIC does not directly give the probability of problems in a grid, but the internal construction of transformers and adjustments of relay operations, for example, play a major role concerning the vulnerability of a system to GIC. To study this aspect is beyond the objectives of the EURISGIC project.

We have shown that the large majority of single site maxima of GIC in 1996-2008 occurred during a few days only, of which 29-30 October 2003 are dominating. A more detailed investigation of GIC in the European high-voltage power grid during these largest storms will be considered in a later paper. Another important topic is to speculate whether a superstorm much larger than any event in 1996-2008 could cause extraordinary high activity in South and Central Europe too. Thomson et al. (2011) and Pulkkinen et al. (2012) have performed statistical studies to assess the magnitude of such rare events, and Ngwira et al. (2013a) investigated 12 severe magnetic storms in 1989-2005. As a recent case study, Baker et al. (2013) considered an extreme solar wind event on 23-24 July 2012 observed by STEREO-A. The solar eruption was not Earthdirected, but Ngwira et al. (2013b) performed a simulation assuming that it would have hit Earth. They found that geoelectric fields could then have been comparable or larger than during the March 1989 and October 2003 storms. Especially, they noted that the geoelectric fields at mid-latitudes could have been larger than reported by Ngwira et al. (2013a). In such a case, most regions of Europe would have experienced a major GIC event.

Acknowledgements. The research leading to these results has received funding from the European Community's Seventh Framework Programme (FP7/2007-2013) under Grant Agreement No. 260330. We thank the large number of institutes providing magnetometer data to the World Data Centre for Geomagnetism (Edinburgh) and INTERMAGNET, and to the IMAGE magnetometer network.

\section{References}

Ádám, A., E. Prácser, and V. Wesztergom, Estimation of the electric resistivity distribution (EURHOM) in the European lithosphere in the frame of the EURISGIC WP2 project, Acta Geod. Geoph. Hung., 47, 377-387, DOI: 10.1556/AGeod.47.2012.4.1, 2012.

Baker, D.N., X. Li, A. Pulkkinen, C.M. Ngwira, M.L. Mays, A.B. Galvin, and K.D.C. Simunac, A major solar eruptive event in July 2012: Defining extreme space weather scenarios, Space Weather, 11, 585-591, DOI: 10.1002/swe.20097, 2013.

Beggan, C.D., D. Beamish, A. Richards, G.S. Kelly, and A.W.P. Thomson, Prediction of extreme geomagnetically induced currents in the UK high-voltage network, Space Weather, 11, 407-419, DOI: 10.1002/swe.20065, 2013.

Liu, C.-M., L.-G. Liu, and R. Pirjola, Geomagnetically induced currents in the high-voltage power grid in China, IEEE Transactions on Power Delivery, 24, 2368-2374,

DOI: 10.1109/TPWRD.2009.2028490, 2009.

Marshall, R., E. Smith, M. Francis, C. Waters, and M. Sciffer, A preliminary risk assessment of the Australian region power network to space weather, Space Weather, 9, S10004,

DOI: 10.1029/2011SW000685, 2011.

Marshall, R.A., M. Dalzell, C.L. Waters, P. Goldthorpe, and E.A. Smith, Geomagnetically induced currents in the New Zealand power network, Space Weather, 10, S08003,

DOI: 10.1029/2012SW000806, 2012.

Minamoto, Y., and Y. Taguchi, Significant decreases in the geomagnetic indices in the ascending phase of Solar Cycle 24, Earth Planets Space, 61, e25-e28, 2009.
Myllys, M., A. Viljanen, Ø.A. Rui, and T.M. Ohnstad, Geomagnetically induced currents in Norway: the northermost high-voltage power grid in the world, J. Space Weather Space Clim., 5, A10, DOI: 10.1051/swsc/2014007, 2014.

Ngwira, C.M., A. Pulkkinen, L.-A. McKinnell, and P.J. Cilliers, Improved modeling of geomagnetically induced currents in the South African power network, Space Weather, 6, S11004, DOI:10.1029/2008SW000408, 2008.

Ngwira, C.M., A. Pulkkinen, F.D. Wilder, and G. Crowley, Extended study of extreme geoelectric field event scenarios for geomagnetically induced current applications, Space Weather, 11, 121131, DOI: 10.1002/swe.20021, 2013a.

Ngwira, C.M., A. Pulkkinen, M. Leila Mays, M.M. Kuznetsova, A.B. Galvin, K. Simunac, D.N. Baker, X. Li, Y. Zheng, and A. Glocer, Simulation of the 23 July 2012 extreme space weather event: What if this extremely rare CME was Earth directed? Space Weather, 11, 671-679, DOI: 10.1002/2013SW000990, 2013b.

Pulkkinen, A., S. Lindahl, A. Viljanen, and R. Pirjola, October 2931, 2003 geomagnetic storm: geomagnetically induced currents and their relation to problems in the Swedish high-voltage power transmission system, Space Weather, 3, S08C03, DOI: 10.1029/2004SW000123, 2005.

Pulkkinen, A., R. Kataoka, S. Watari, and M. Ichiki, Modeling geomagnetically induced currents in Hokkaido, Japan, Adv. Space Res., 46, 1087-1093, DOI: 10.1016/j.asr.2010.05.024, 2010.

Pulkkinen, A., E. Bernabeu, J. Eichner, C. Beggan, and A.W.P. Thomson, Generation of 100-year geomagnetically induced current scenarios, Space Weather, 10, S04003, DOI: 10.1029/2011SW000750, 2012.

Thomson, A.W.P., A.J. McKay, E. Clarke, and S.J. Reay, Surface electric fields, geomagnetically induced currents in the Scottish Power grid during the 30 October 2003 geomagnetic storm, Space Weather, 3, S11002, DOI: 10.1029/2005SW000156, 2005.

Thomson, A., E. Dawson, and S. Reay, Quantifying extreme behaviour in geomagnetic activity, Space Weather, 9, S10001, DOI: 10.1029/2011SW000696, 2011.

Torta, J.M., L. Serrano, J.R. Regué, A.M. Sánchez, and E. Roldán, Geomagnetically induced currents in a power grid of northeastern Spain, Space Weather, 10, S06002,

DOI: 10.1029/2012SW000793, 2012.

Trivedi, N.B., I. Vitorello, W. Kabata, S.L.G. Dutra, A.L. Padilha, et al., Geomagnetically induced currents in an electric power transmission system at low latitudes in Brazil: a case study, Space Weather, 5, S04004, DOI: 10.1029/2006SW000282, 2007.

Viljanen, A., European project to improve models of geomagnetically induced currents, Space Weather, 9, S07007, DOI: 10.1029/2011SW000680, 2011.

Viljanen, A., A. Koistinen, K. Pajunpää, R. Pirjola, P. Posio, and A. Pulkkinen, Recordings of geomagnetically induced currents in the finnish natural gas pipeline - summary of an 11-year period, Geophys. J., 46, 59-67, 2010.

Viljanen, A., H. Nevanlinna, K. Pajunpää, and A. Pulkkinen, Time derivative of the horizontal geomagnetic field as an activity indicator, Ann. Geophys., 19, 1107-1118, DOI: 10.5194/angeo-19-1107-2001, 2001.

Viljanen, A., R. Pirjola, M. Wik, A. Ádám, E. Prácser, Y. Sakharov, and J. Katkalov, Continental scale modelling of geomagnetically induced currents, J. Space Weather Space Clim., 2, A17, DOI: 10.1051/swsc/2012017, 2012.

Viljanen, A., R. Pirjola, E. Prácser, S. Ahmadzai, and V. Singh, Geomagnetically induced currents in Europe: characteristics based on a local power grid model, Space Weather, 11, 575-584, DOI: 10.1002/swe.20098, 2013.

Viljanen, A., A. Pulkkinen, R. Pirjola, K. Pajunpää, P. Posio, and A. Koistinen, Recordings of geomagnetically induced currents and a nowcasting service of the Finnish natural gas pipeline system, Space Weather, 4, S10004, DOI: 10.1029/2006SW000234, 2006.

Viljanen, A., and E. Tanskanen, Climatology of rapid geomagnetic variations at high latitudes over two solar cycles, Ann. Geophys., 29, 1783-1792, DOI: 10.5194/angeo-29-1783-2011, 2011. 
A. Viljanen et al.: Geomagnetically induced currents in Europe

Watari, S., M. Kunitake, K. Kitamura, T. Hori, T. Kikuchi, et al., Measurements of geomagnetically induced current in a power grid in Hokkaido, Japan, Space Weather, 7, S03002,

DOI: 10.1029/2008SW000417, 2009.

Weigel, R.S., A.J. Klimas, and D. Vassiliadis, Solar wind coupling to and predictability of ground magnetic fields and their time derivatives, J. Geophys. Res., 108 (A7), 1298, DOI: 10.1029/2002JA009627, 2003
Wik, M., A. Viljanen, R. Pirjola, A. Pulkkinen, P. Wintoft, and H. Lundstedt, Calculation of geomagnetically induced currents in the $400 \mathrm{kv}$ power grid in Southern Sweden, Space Weather, 6, S07005, DOI: 10.1029/2007SW000343, 2008.

Wik, M., R. Pirjola, H. Lundstedt, A. Viljanen, P. Wintoft, and A. Pulkkinen, Space weather events in July 1982 and October 2003 and the effects of geomagnetically induced currents on Swedish technical systems, Ann. Geophys., 27, 1775-1787, DOI: 10.5194/angeo-27-1775-2009, 2009.

Cite this article as: Viljanen A, Pirjola R, Prácser E, Katkalov J \& Wik M, et al.: Geomagnetically induced currents in Europe. J. Space Weather Space Clim., 2014, 4, A09. 